\title{
An Intelligent Model for Assessment of Cough in Covid-19 Infected Patients based on Sound to Predict their Clinical Criticality using XGB Algorithm
}

\author{
Dr. Jyothi N.M ${ }^{1}$ \\ ${ }^{1}$ K L University, India, jyothiarunkr@ gmail.com
}

\begin{abstract}
Cough is a one of the major symptoms of COVID 19 infected patients. This research study underlines automatic, objective, and reliable assessment of cough events to predict severity of infection in patients suffering from novel Corona Virus. The system is self-learning and thus intelligent. In this research paper, a brief survey of an audio-based cough monitoring systems, cough detection and then illustrated the cough sound generating principle. Clinical parameters such as cough frequency, intensity of coughing, and acoustic properties of cough sound, were also analyzed in this paper. Steps of Cough sound processing are also considered. Brief studies of cough sound processing algorithms are also made. Finally, XGB algorithm is chosen as the predictor, due to its superb classification and feature selection ability. Finally, end result of clinical criticality of Covid-19 infected patients is predicted. However for the research purpose, the cough sound recording of Covid-19 cough data set is created.. The trained model was used to prediction. The predicted data is found to be accurate.
\end{abstract}

Key words : Covid 19, STx, sound preprocessing, XGB

\section{INTRODUCTION}

In this research paper the assessment of cough severity is measured in order to predict the criticality of the Covid 19 infected person. Measure of cough frequency is the measure the severity of cough and hence assesses criticality of the patient. .[1]. The cough sound recording of the patients is obtained and after preprocessing the cough signal is detected and counted and measure of frequency of the cough sound is used to assess the criticality of the patient and predict as low, medium or high and thus helping the patients to get better treatment and helping doctors to save patient's life. For the purpose of classification of cough and prediction of patient's criticality powerful XGB algorithm is applied.

\section{COVID 19 AND COUGH}

Novel Corona virus has impacted lives of people in many ways. Symptoms of the virus are fever, headache, frequent wheezing, coughing, trouble in breathing and shortness of breath to name just few.. COVID-19 broke out in Wuhan city of China in December 2019, and since then it has spread all over the world at a drastic rate and immediately become a pandemic as declared by World Health Organization..It is causing serious public health problem all over the world.. Till now no proper medicine against COVID-19 is being invented by any country. However, for the person infected with COVID-19, his clinical criticality and their mortality risk attached with infection could potentially be predicted before the patients enter critical stage. This can help doctors with early identification and intervention andgive better treatment and, thus potentially reducing mortality rate of the patients..

There have been three main ways to record cough. One is based on the airflow measurement at the mouth to obtain the flow dynamics of cough $[3,4]$.. The second is based on the movement of the chest. For example, some researchers [5] invented an accelerometer-based system that used an accelerometer placed at the volunteer's chest wall to record cough events. Third method is measurement of cough sounds. The last method is more universal because of advances in computer technology and the availability of portable digital sound recording devices [6]. As the patient is already tested positive for COVID-19 the physical characteristic of the cough is characterized as dry. This research paper focuses on audio-based model to assess and predict the patient's clinical criticality using cough sound signals by applying XGB.

\subsection{Cough Patterns Acoustic Properties of Cough Sound}

The typical cough sound is usually divided into three stages. First stage is an explosive expiration due to the glottis suddenly opening. Second stage is the intermediate phase with the attenuation of cough sounds, and the third stage is the voiced phase due to the closing of the vocal cord. Cough patterns and the quality of a patient's cough sound may reflect useful information about their condition. There is relationship between cough patterns (or acoustic properties of cough 
Jyothi N.M, International Journal of Emerging Trends in Engineering Research, 9(1), January 2021, 1 - 5

sounds) and the criticality of the illness-triggering cough. In this research paper illness is considered as COVID-19 infection. Cough Frequency of a patient measures the energy level with which the cough sound is generated and Cough count is helpful in measuring the severity of the patient. Current chronic cough frequency monitors usually use explosive cough sounds to evaluate cough frequency [7]. 24-hour cough frequency is proved correct and effective in a longitudinal observational study of 33 healthy subjects with acute cough [8]. 4-hour cough frequency is found to be responsive to improvements in cough severity following trials of therapy in 100 patients with chronic cough [9].Cough Intensity is in Cough sound is a potential measure of the intensity of coughs. The intensity of cough is based on peak cough flow rate, esophageal pressure, and gastric pressure .These are important and relevant measures of cough intensity in patients [10,11], and these indexes can be calculated for a time window with a duration of 0.5 second from one set of stage 1, the explosive phase of cough sound [11].

\section{OBTAINING COUGH SOUND}

The first step in the experimental setup is obtaining the cough sound of the patients. Cough signals are captured using microphone/mobile phone/any recording system. As research deals with covid 19 related cough, it is advisable to get the cough sound recorded by the patient himself through his mobile phone using built in sound recording software provided in the mobile phone or recording could be done by health workers near to him wearing personal protective kit as virus is highly contagious.. The recorded audio file in the mobile is received from patient's mobile via WhatsApp/email analog with the patient's id and name, date and time. Minimum 10 mins recording per day is considered for experimental purpose to predict patient's criticality. However, it can be taken two times per day once in morning and then in evening for a duration of ten to twenty minutes for better accuracy. As it build very huge data set of cough sound recording. This is possible for commercial purpose. But however, in this experimental model only small scale data is considered for twenty patents.

\subsection{STx}

The audio file received by the patient is saved in the system along with patient's details(Patient ID, Date, Time of record and duration). The sound file is converted to .wav format for the purpose of further processing The wav file is fed into STx- Sound Tools Extended (STx) intelligent sound processing software. The software is actively being developed at the Acoustics Research Institute in Vienna, and is used in a variety of acoustic fields around the world. It is an acoustic speech and signal processing application. It provides tools to analyze, visualize, segment, and annotate wave files. The converted cough sound .wav file is divided into several segments at an interval of 5 seconds each in STx, so that each
$5 \mathrm{sec}$ frame can be analyzed for cough signal as shown in Figure 1.

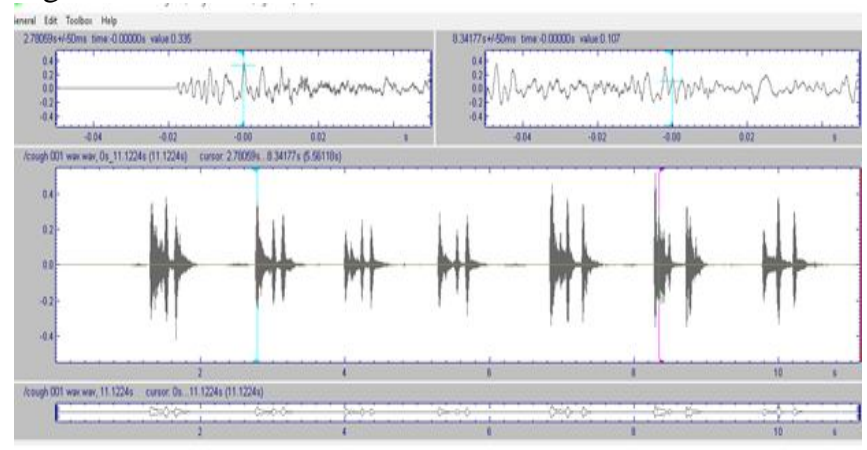

Figure 1: The wave format of the cough sound signal captured from patient's cough sound recording

\subsection{Cough Sound processing}

Preprocessing steps involved in cough detection noise reduction, silence removal, differentiation from patient sounds, especially speech, laughing, and sneezing

i. Sampling is a process of converting an analog signal to a digital form for making computer to store these cough signals in the memory. Sampling rate defines the frequency at which the sampling occurs for the purpose of end point detection. Sampling rate is chosen as 1 second.

ii. Noise removal is done using filtering mechanism provided by the Stx software. And silence part has low or zero energy level and it is also removed by feeding the file into Audacity Software as Audacity > Effect > Truncate Silence > adjust as desired.. Or it can be removed manually also.

iii. End point detection actually detects the cough signal which is usually explosive in nature. Each segment can be determined by a beginning and endpoints. Cough has explosive sounds. The energy level of cough sound is high on the raising edge and it has bursting frequency. The distinguishing characteristics between these two points based on energy levels define the energy based determination. The accuracy of the cough recognition is dependent purely on how efficiently the end point detection takes place. As shown in Figure 2..

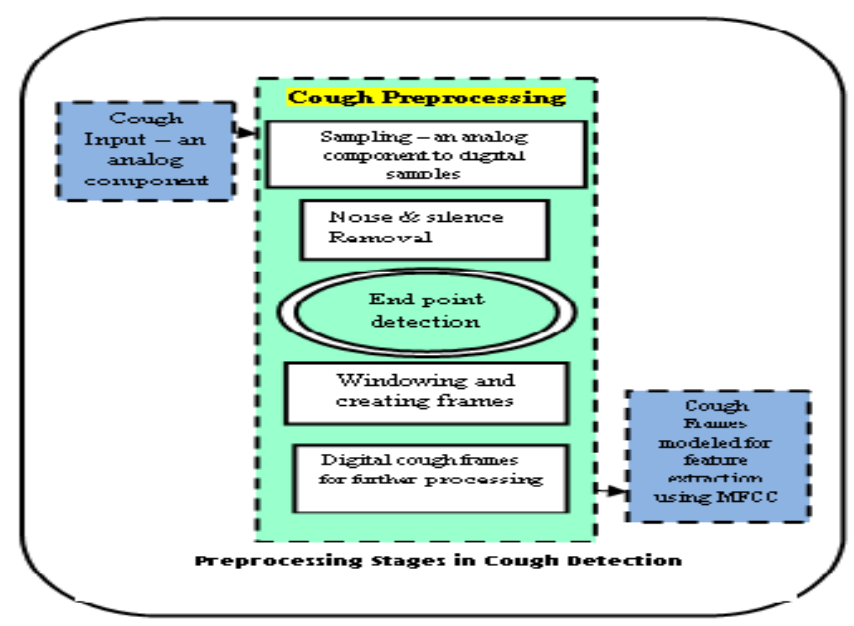

Figure 2 : Steps involved in cough sound preprocessing 
Jyothi N.M, International Journal of Emerging Trends in Engineering Research, 9(1), January 2021, 1 - 5

iv. Windowing and Frame formation - The standard technique used for windowing and framing is Mel Frequency Cepstral Coefficient (MFCC) method. MFCC aims to produce windowing for a cough waveform sampled at definite time interval. Each waveform indicates continuous ripples of sound structure. MFCC ensures that this continuous waveform is divided at finite time interval and then each sub division of the wave put in a boundary called window. In order to separate the continuous waveform into discontinuous, Fourier Transformation whilst Discrete Fourier Transformation (DST) have to be used. DST is a process of separating ' $n$ ' waves at a definite sample time interval ' $t$ ' i.e. a process of converting the continuous sequence of data into a fixed sequence of data. Let $\mathrm{S}(\mathrm{t})$ represent the continuous waveform generated from a given input. The ' $n$ ' instant of wave samples be represented as $S|0|, S|1|, \ldots S|n-1|$

Therefore the DST can be written as shown in equation [1],

$S(j w)=\int_{-\infty}^{\infty} S(t) e^{-j w t} d t$

After integrating equation [1] with a constant say ' $\mathrm{k}$ ', we arrive at equation [2],

$S(j w)=\sum_{k=0}^{n-1} f[k] e^{-j w k t}$

Now the frames we got consist of cough frames and thus cough sound is successfully detected as shown Figure. 3. The cough sound frames can be modeled for feature extraction using MFCC from time domain signal as explained above and then further inputting them into a model classifier.

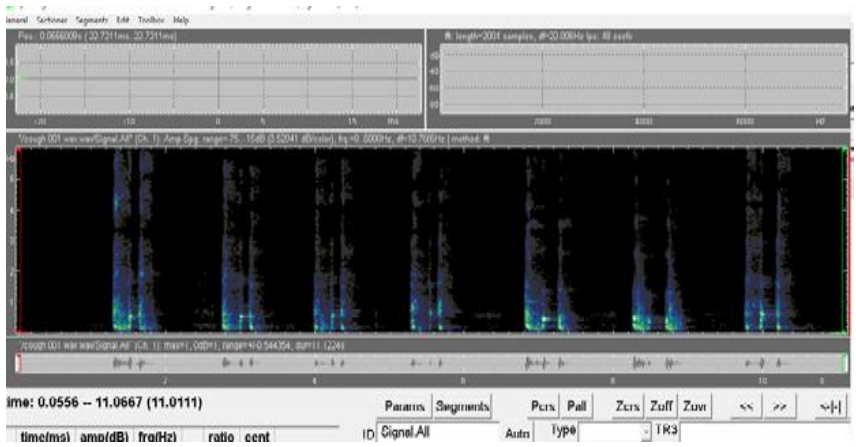

Figure 3: Windowing and Framing

\section{COUGH SOUND ASSESSMENT AND BUILDING TRAINING DATA}

Second part of the research consists of assessment of cough by classification and by count. This builds initial training data required for the purpose of the model. And then applying XGB algorithm on test input data for classification and prediction. XGB to help in predicting criticality of patients,

\subsection{Steps involved in classification and building training data}

i. The patient's cough audio file(.wav) of 10 minutes duration for that day is input into STx.

ii. The frequency level of every cough sound signal is obtained from STX.

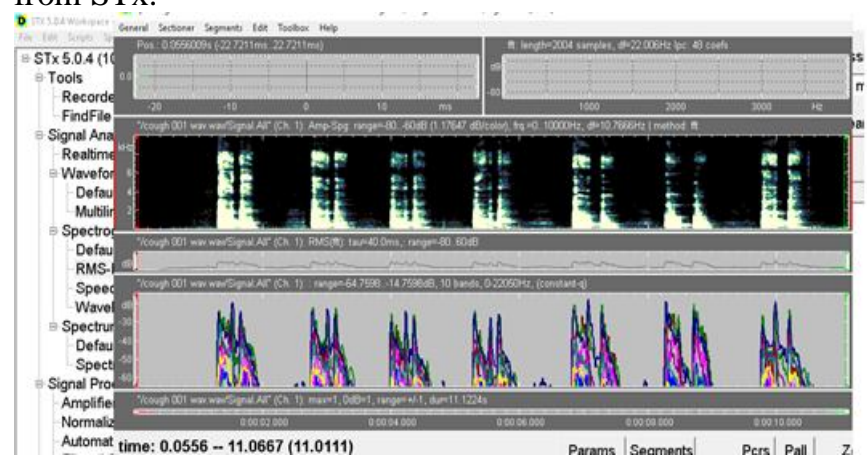

Figure. 4: Peak cough frequency

iii. The frequencies of framed cough signals are stored in the data base along with the patient id, date, time stamp, cough frequency and cough count

iv. Algorithm : Classification of cough

Step1.Cough signal is labeled as LOW [numeric value 1] if cough count $<=30$ and avg frequency level of cough signals is $<=300 \mathrm{~Hz}$

(cough sound recording of 10 mins on that particular date can be taken)

Step2. Cough signal is labeled as MED [numeric value 2] if cough count $>30 \& \&$ cough Count $<=100$ and average frequency level of cough is $>300 \mathrm{~Hz}$ and $<600 \mathrm{~Hz}$

(cough sound recording of 10 mins on that particular date can be taken)

Step 3:Cough signal is labeled as HIGH [numeric value 3] if cough count $>100$ and avg frequency level of cough is $<600 \mathrm{~Hz}$ (cough sound recording of $10 \mathrm{mins}$ on that particular date can be taken)

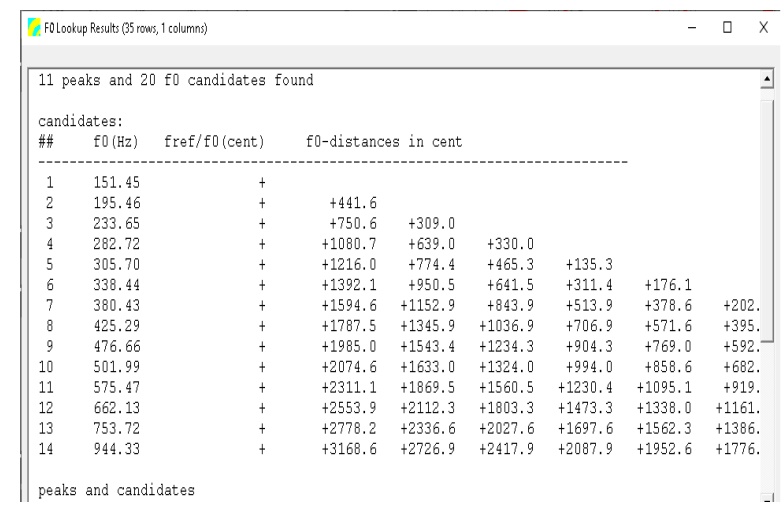

Figure 5: Sample Cough sound frequency (f0) 
The Figure 5 shows the sample of the cough frequency recorded by a patient. Using Stx software the frequencies of the cough signal are extracted. Simple code can be written using python language to categorize the cough frequency as low/medium/high initially to build training data. Cough frequency is shown in HZ. Stx sorts the frequencies by default. Cough frequency between less than $300 \mathrm{~Hz}$ is labeled as LOW(1)Cough frequency between 301 to 600 is labeled as $\mathrm{MED}(2)$ and cough frequency above $601 \mathrm{~Hz}$ is labeled as $\mathrm{HIGH}(3)$. Using simple if else code labeling is done.

The training data set is built and stored for future use. The categorized (labeled) data of a patient is stored in separate table or .csv file as training data to be used by XGB algorithm. The process is repeated every day for all the patients until sufficient training data is built for a model .For the experiment purpose data set consisting of 10000 labeled cough sound frequency from 20 patients is built and stored for the first five days. So that , next five days the obtained cough signal can be used as test data for learning purpose by the XGB algorithm.

\subsection{XGB (Extreme Gradient Boosting)}

Extreme Gradient Boosting is a popular implementation of Gradient Boosting because of its speed and performance. Internally, XGBoost models represent all problems as a regression predictive modeling problem that only takes numerical values as input. XGBoost is used for supervised learning problems, where we use the training data (with multiple features) xi to predict a target variable yi.

The model in supervised learning usually refers to the mathematical structure of by which the prediction yi is made from the input xi. The prediction value can have different interpretations, depending on the task, i.e., regression or classification. With judicious choices for yi, we may express a variety of tasks, such as regression, classification, and ranking. The task of training the model amounts to finding the best parameters that best fit the training data xi and labels yi.

\subsection{Predicting patient's criticality by applying XGB}

Input the test data consisting of cough frequncies of a patients collected that needs to be predicted.The XGB algorithm checks the training data and learns and predicts the criticality as high(3) or medium(2) or low(1) depending on the majority count of the category of the frequencies of cough signal that are labeled. When new cough data is input, the XGB algorithm checks with the existing training data and classifies the data as Low, Med, High.The majority count of the label is taken as patient's criticality. If there is tie in the label count, the worst critical label is considered for that day. Example, tie between Low and High means criticality is conserved as High an similarly between Low and Med, criticality is considered as Med and test is continued for next day.

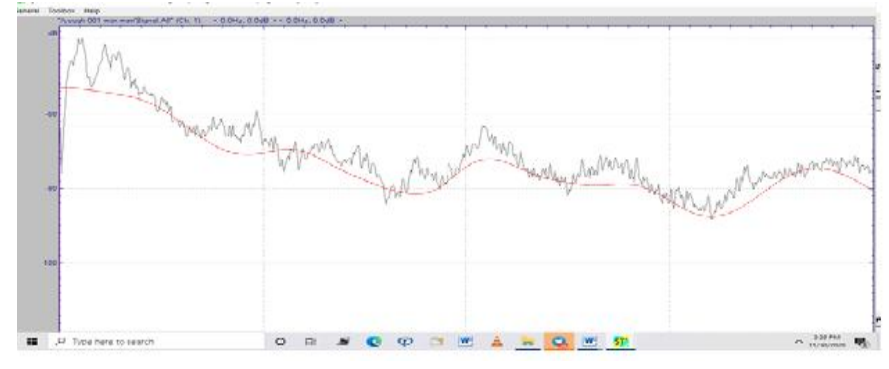

Figure 6 : Cough map of a patient's recorded cough sound

\section{EXPERIMENTAL SETUP}

\section{Steps for Applying XGB}

i. Install XGBoost for use with Python.

ii. Define the Problem and download dataset.

iii. Load and prepare Cough data.(X)

iv. Train XGBoost model.

v. Make predictions and evaluate $\operatorname{model}(\mathrm{Y})$.

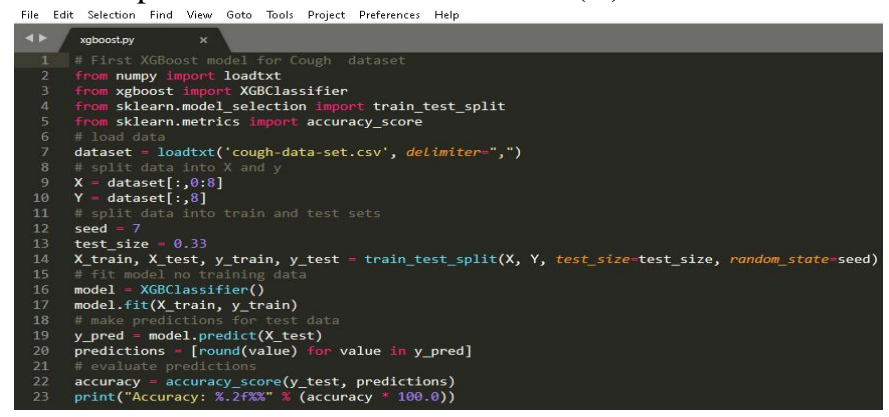

Figure 7: Sample code to implement XGB

Experimental result gave $87.75 \%$ accuracy for classification and predictions of the patient's criticality is also made with accuracy of $87 \%$.. As per the survey observed, till now no research is carried out using XGB algorithm on audio based cough sound analysis and also in detecting the criticality of covid 19 patients by cough sound signal to assess and make predictions using XGB. This research is first of its kind. The XGB is a powerful Machine Learning algorithm which could be effectively used in classification and prediction. The prediction accuracy could be increased still further by building more records of training dataset for Covid 19 infected cough sound signals. The model in this research paper helps in predicting the critical level of the patient's health and helping the physicians to decide and plan in advance to give the advanced treatment according to the criticality label obtained by the model and thus helping in saving the precious lives of the people affected by Covid 19 virus.

\section{CONCLUSION}

As cough is one of the major symptoms of Covid 19 infection, It can be used as one of the powerful parameters in determining the patient's criticality and XG Boost Algorithm is one of the powerful algorithms in Machine Learning and it's power can be used in training the cough test data and 
Jyothi N.M, International Journal of Emerging Trends in Engineering Research, 9(1), January 2021, 1 - 5

classifying cough frequency. Prediction of criticality is made based on the majority of the labels count for a patient based on daily collected cough data and it proved be clinically correct and accurate. The model could be used in any prediction of patient's criticality where cough plays major role as it reflects the respiratory health of the person and could be commercially deployed.

\section{REFERENCES}

1. H. A. Bickerman and S. E. Itkin, "The effect of a new bronchodilator aerosol on the air flow dynamics of the maximal voluntary cough of patients with bronchial asthma and pulmonary emphysema," Journal of Chronic Diseases, vol. 8, no. 5, pp. 629-636, 1958.

2. W. T. Goldsmith, A. M. Mahmoud, J. S. Reynolds et al., "A system for recording high fidelity cough sound and airflow characteristics," Annals of Biomedical Engineering, vol. 38, no. 2, pp. 469-477, 2010.

3. S. Ren, Y. Shi, M. Cai, and W. Xu, "Influence of secretion on airflow dynamics of mechanical ventilated respiratory system," IEEE/ACM Transactions on Computational Biology and Bioinformatics, vol. PP, no. 99, p. 1, 2017.

4. Y. Hu, E. G. Kim, G. Cao, S. Liu, and Y. Xu, "Physiological acoustic sensing based on accelerometers: a survey for mobile healthcare," Annals of Biomedical Engineering, vol. 42, no. 11, pp. 2264-2277, 2014.

5. E. C. Larson, T. J. Lee, S. Liu, M. Rosenfeld, and S. N. Patel, "Accurate and privacy preserving cough sensing using a low-cost microphone," in Proceedings of the 13th International Conference on Ubiquitous Computing - UbiComp '11, Beijing, China, September, 2011.

6. Y. Shi, B. Zhang, M. Cai, and X. D. Zhang, "Numerical simulation of volume-controlled mechanical ventilated respiratory system with 2 different lungs," International Journal for Numerical Methods in Biomedical Engineering, vol. 33, no. 9, 2016.

7. K. K. Lee, S. Matos, D. H. Evans, P. White, I. D. Pavord, and S. S. Birring, "A longitudinal assessment of acute cough," American Journal of Respiratory and Critical Care Medicine, vol. 187, no. 9, pp. 991-997, 2013.

8. K. K. Lee, A. Savani, S. Matos, D. H. Evans, I. D. Pavord, and S. S. Birring, "Four-hour cough frequency monitoring in chronic cough," Chest, vol. 142, no. 5, pp. 1237-1243, 2012

9. L. Pavesi, S. Subburaj, and K. Portershaw, "Application and validation of a computerized cough acquisition system for objective monitoring of acute cough: a meta-analysis," Chest, vol. 120, no. 4, pp. 1121-1128, 2001.

10. K. K. Lee, S. Matos, K. Ward, E. Raywood, D. H. Evans, J. Moxham et al., "P158 cough sound intensity: the development of a novel measure of cough severity," Thorax, vol. 67, Supplement 2, pp. A130.3-A1A131, 2012.

11. https://xgboost.readthedocs.io/en/latest/tutorials/model.html 